\title{
An Experimental Investigation on the Relationship between MS Frequency Response and Coal and Gas Outburst
}

\author{
Quanjie Zhu $\left(\mathbb{D},{ }^{1,2}\right.$ Qingsong Li, ${ }^{3}$ Yu Feng, ${ }^{4}$ Xianwei Heng, ${ }^{3}$ Shaoquan Li, ${ }^{3}$ and Tao Yang ${ }^{1}$ \\ ${ }^{1}$ Hebei State Key Laboratory of Mine Disaster Prevention, North China Institute of Science and Technology, Sanhe 065201, China \\ ${ }^{2}$ Hubei Key Laboratory for Efficient Utilization and Agglomeration of Metallurgic Mineral Resources, \\ Wuhan University of Science and Technology, Wuhan 430081, China \\ ${ }^{3}$ Guizhou Mine Safety Scientific Research Institute, Guiyang 550025, China \\ ${ }^{4}$ Department of Civil Engineering, University of Toronto, Ontario, Canada M5S 1A4
}

Correspondence should be addressed to Quanjie Zhu; youyicun2008@gmail.com

Received 27 December 2017; Revised 5 March 2018; Accepted 25 March 2018; Published 26 June 2018

Academic Editor: Longjun Dong

Copyright (C) 2018 Quanjie Zhu et al. This is an open access article distributed under the Creative Commons Attribution License, which permits unrestricted use, distribution, and reproduction in any medium, provided the original work is properly cited.

Microseismic (MS) frequency response is an important part of high-efficiency data mining to achieve the aim of coal and gas outburst (CGOB) early warning. Based on the variation pattern of acoustic emission (AE) signal in the coal failure process, the experimental characteristics of MS activity and typical signals CGOB were obtained in this study. First, the AE behavior of coal failure experiment was studied, and an explanation of laws was provided as follows: the fracture behavior of coal sample exhibits certain characteristics of $\mathrm{AE}$ response in terms of $\mathrm{AE}$ event count, signal amplitude, and frequency; each stage has its own physical meaning during the process of loading test. Based on these laws, CGOB experiments were carried out using a large CGOB physical simulation system with a MS monitoring system. Notching filter and wavelet packet transform technique were used in the denoising and feature extraction of six typical MS events (signals). The features of each stage, including the time-frequency domain, were extracted and quantitatively expressed. We finally arrive at the following conclusions: (1) CGOB exhibits significantly periodic characteristics, and each CGOB stage corresponds to the significant response characteristics of MS. CGOB presents varying characteristics, such as "valley-peaks-valley". (2) From the incubation stage to happen stage of outburst, the spectrum significantly moved from extremely low frequency (100-200 Hz) to high-frequency band (approach to $1600 \mathrm{~Hz}$ ). During the residual stage, MS frequency manifested the concentration distribution $(50 \mathrm{~Hz})$ and offered the advantage of energy concentration. (3) The phenomenon of signal energy also shows the trend of energy transform low to high and to low modes along with the process. Signals total energy distribution $(42.81 \%, 1,437.5-1,812.5 \mathrm{~Hz})$ in the happen stage are markedly larger than those of events in incubation stage (7.01\%) and residual stage (1.44\%). The methodology presented in this paper for CGOB signal analysis provides a new method to obtain MS response precursor and predict CGOB disaster. This approach can be useful for rockburst anticipation and control during mining in gas and highly stressed coal mines.

\section{Introduction}

Coal and gas outburst (CGOB) disasters are characterized by a complicated mechanism, sudden occurrence, and massive harm [1-4]. CGOB disasters not only affect normal operations of mining but also seriously restrict mine safety and efficient production. To date, existing parameters and methods are limited in making accurate predictions and fail to satisfy the requirements of mining safety; Figure 1 shows a typical unforecasted CGOB accident that occurred in Guizhou Province. Therefore, prediction and prevention studies should be related to reduce casualties and economic losses caused by CGOB disasters.

CGOBs are controlled by multiple factors, such as stress, gas, physical, and mechanical properties of coal [512]. Capturing characteristic information before the disaster occurs is crucial to achieve accurate and timely warning. According to the investigation and analysis of accidents, CGOB is commonly accompanied by precursor information $[13,14]$, such as drilling jamming, gas abnormal effusing, temperature changes of coal body, gas pressure increase, and hissing noise released from coal body. Many researchers 


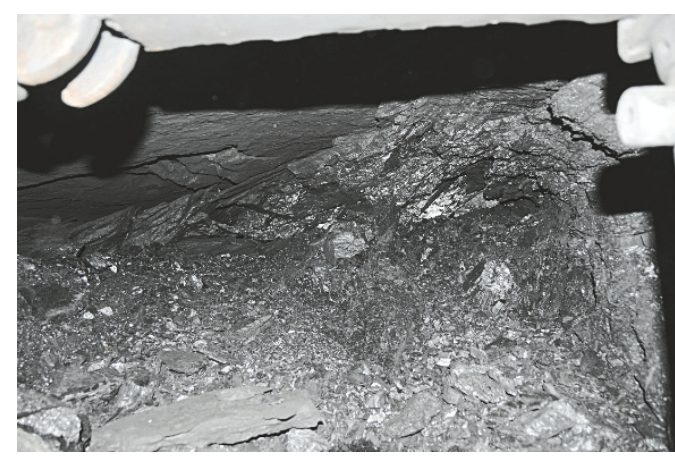

(a) Outburst and piling up seam

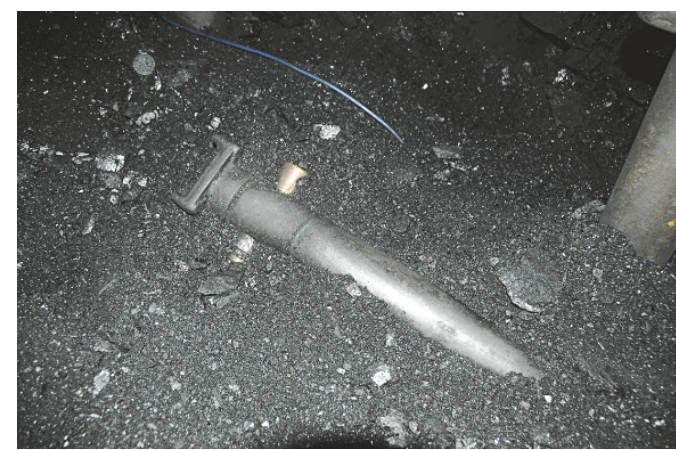

(b) Dumping and buried single hydraulic props

FIGURE 1: Disaster of CGOB in Guizhou Province, China.

suggested using gas pressure, coal bed stresses, and methane concentration parameters based on these characteristic pieces of information to predict CGOB disasters. The main contactless monitoring and early warning methods to predict the CGOB disasters include electromagnetic radiation (EMR) method [15-18], acoustic emission (AE) method [19, 20], and microseismic (MS) method [21]. Compared with AE and EMR, MS inversion technology exhibits advantages in source inversions, monitoring range, and signal connotation aspects over a large size monitoring range [22-24]. MS method has been widely used for mining disaster prediction, such as CGOB, because onsite monitoring and laboratory research indicate that microfracturing is a typical precursor of dynamic disaster [25-27].

MS signal analysis, processing, and feature mining are key to realize accurate and early warning, which is still an urgent problem. In practice, the characteristics of MS event, signal recognition [28], and precise positioning [29, 30] are the important parts of signal process and analysis. The related theory of signal has been advanced for many years. At present, certain methods such as time-domain analysis, frequencydomain analysis, and time-frequency domain analyses have been conducted to capture signal characteristics. Among these methods, time-frequency analysis method is widely used in blasting, machinery, and the other areas of signal analysis, especially seismic signal processing [31-33]. Typical methods, such as Fourier transform, continuous wavelet transform, S transform, Wigner-Ville distribution, and Hilbert transform (HHT), are based on signal frequency analysis with primary short-time Fourier transform [34], wavelet transform [35], discrete wavelet transform (DWT) [36], and wavelet packet transform [37]. Time frequency analysis is a complex signal processing method that can be used for constituent and transient nonstationary MS signals. Kang (2010) analyzed the time frequency of AE signals from rock damage and obtained time-delay estimation in a single band, which resulted in a damage localization with enhanced accuracy [38]. Zhu (2012) also conducted research on wavelet packet energy to classify blasting and MS signals [39]. Guizhou Coal Mine Design and Research Institute has investigated MS signal analysis and prediction of CGOB in recent years and obtained significant results through a series of laboratory experiments $[40,41]$.
This paper aims to find an effective feature for describing the process changes of CGOB and explore a new approach to disaster prediction. In general, this study can be primarily divided into two parts: uniaxial compression test (UCT) of coal specimens containing gas (CSCG) and large-scale CGOB test in the laboratory. In previous experiments [40, 41], we found that the main frequency of AE signal changes with the coal specimen failure process. On the basis of this result, we present a novel method to analyze the weak and nonstationary transient characteristics of MS signals during CGOB. Through analysis and processing, we observed that AE or MS signal frequency was regularly changed during CGOB. We describe the problem in detail and demonstrate how to find a solution.

\section{AE Behavior of Coal Failure Process}

2.1. AE Dynamic Response under UCT. Previous studies show that the failure process of coal-rock combined body exhibits apparent AE characteristics that are significant when the specimen is initially damaged, yielded, and destroyed [42-44]. The AE characteristics in UCT with different gas pressures should be understood and interpreted to confirm the coal and rock fracture. Therefore, we performed the UCT study on AE characteristics of coal failure with coal samples stored in a gas pressure environment before the CGOB experiment. The experimental study on the mechanical properties of coal containing methane was performed on raw coal samples in a coal mine located in Guizhou Province, China. AE system choice of high precision equipment from PAC (Physical Acoustic Corporation), the parameters of $\mathrm{AE}$ device were set as follows: main amplifier, $40 \mathrm{~Hz}$; threshold value, $35 \mathrm{db}$; sensor resonant frequency, 20-400 $\mathrm{kHz}$; sample frequency, $1 \mathrm{MHz}$ (more details about this system and parameters can be seen in [43-45]). Figure 2 clearly shows that the AE event count changes with loading stress in different stages. During the laboratory experiment, AE signals occurred along with uniaxial loading at every stage, and AE space-time evolution corresponded to the complete stress-strain curves. Clearly, the fracture behavior of coal sample exhibits certain characteristics, and each stage denotes its own physical meaning during loading test. After a gap, the stage of specimen failure arrives. The count of 

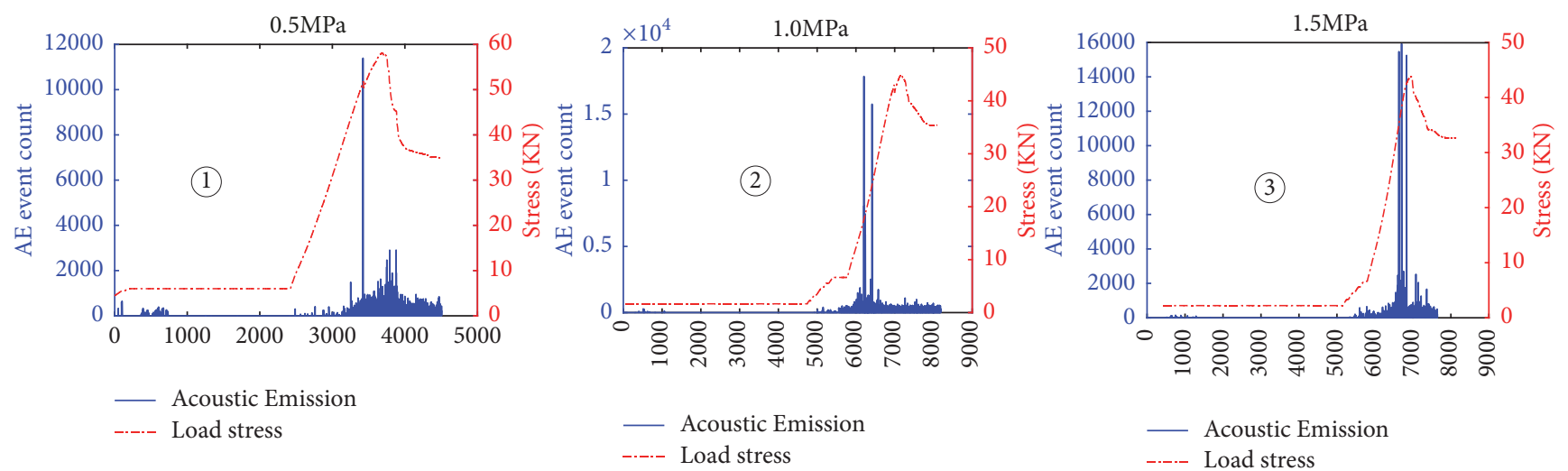

FigURE 2: AE event count and loading stress for different gas pressure levels.
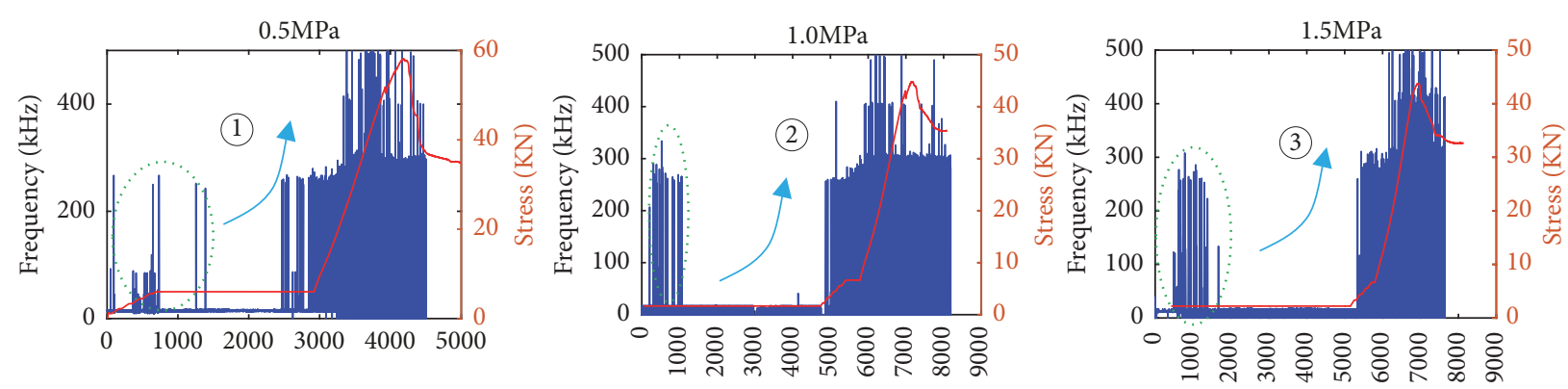

FIGURE 3: Relationship curves of stress-time-AE signal peak frequency.

AE event increases sharply along with the loading stress and then reaches a maximum value. Afterward, the process is completed and enters a quiet period.

2.2. Peak Frequency Variation of AE Signal. In addition, several new conclusions can be defined as an indications or precursors for the CSCG to sprout and develop during loading. As shown in Figure 3, the frequency of AE signals shows an apparent periodic pattern. Sporadic AE responses with high frequency $(>200 \mathrm{kHz})$ appeared in the initial period. The process then developed into a brief period of "blank." When the signal starts to become unsteady, the signal frequency presented a sharp increase from $20 \mathrm{kHz}$ to $500 \mathrm{kHz}$ and remained at high-frequency levels for a short time until the coal ultimately failed. Three specimens with different gas pressure levels displayed similar frequency response patterns, most of which depicted frequency changes from sporadic, blank to shoot up, and from low to high frequency. Our results gave agreement with the corresponding result reported by JI (2015) [46], in which the basic AE characteristics in the low and high frequencies are similar during failure matching the mechanical properties. Thus, signal frequency regularly changes in the cracking process of rock and coal.

The process of UCT exhibits certain regularity of AE response in terms $\mathrm{AE}$ event count, signal amplitude, and frequency. This finding is followed by a description of CGOB laboratory and a detailed presentation of how to quantitatively represent the MS signal and apply it in predicting hazards. In the subsequent sections, we describe the experiment on CGOB disaster to verify whether the result is the same.

\section{CGOB Experiment}

In this section, we introduce the CGOB experimental apparatus and scheme. The experiments were completed on December 25, 2011. Experiment phenomena, especially the MS dynamic response of the experiment, are shown in the following description.

3.1. Experimental Apparatus and Scheme. CGOB experiments were carried out by means of a large CGOB physical simulation system, which was developed independently by Chongqing University (see [7] for more details about this system). The simulation system includes three functional parts (see Figure 4): model steel structure (including sample box), loading device and air ejecting device (including gas compressor), and external monitoring equipment (such as gas pressure and temperature). MS equipment from Engineering Seismology Group, Canada Inc. (ESG), was one of the external equipment used to record the MS vibration in realtime dynamic monitoring during the total CGOB process. The parameters of MS monitoring device were set as follows: sampling frequency, $4,000 \mathrm{~Hz}$; continuous-acquisition buffer length, $15 \mathrm{~min}$ with a subsequent STA/LTA for picking up and intercepting the events; speed sensor frequency, $50-5 \mathrm{kHz}$ with a sensitivity of $30 \mathrm{~V} / \mathrm{g}$; acquisition frequency, $0-2,000$ $\mathrm{Hz}$. 


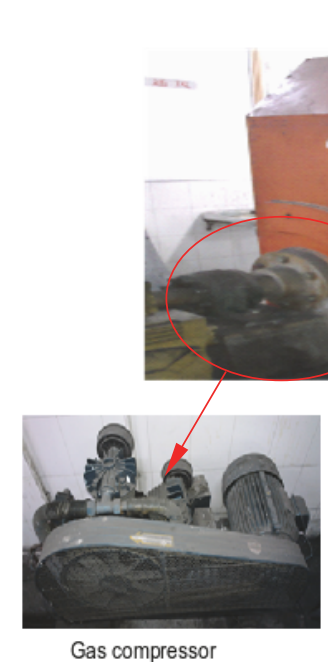

(b)

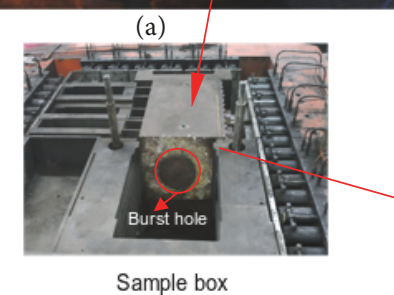

(c)

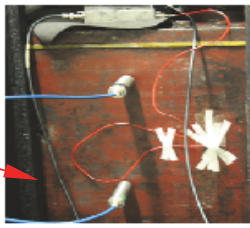

Installation of sensors

(d)

FIGURE 4: Analog simulation system of coal and gas outburst (ASSCGO): (a) steel experimental structure, (b) gas compressor, (c) sample box, and (d) installation of MS sensors.

TABLE 1: Experimental parameters and result.

\begin{tabular}{|c|c|c|c|c|}
\hline Items & & Content & & Result \\
\hline \multirow{4}{*}{ Key parameters } & 1 & Gas content & & $20.125 \mathrm{~m}^{3} / \mathrm{t}$ \\
\hline & 2 & Gas pressure & & $1.41 \mathrm{MPa}$ \\
\hline & 3 & \multirow{2}{*}{ Description of thrown body } & Shape & Cylinder \\
\hline & 4 & & Size & None recorded \\
\hline \multirow{4}{*}{ Key moments } & 1 & Beginning time & & $15: 30: 50$ \\
\hline & 2 & Shutting time & & $16: 29: 00$ \\
\hline & 3 & Preparation time & & $17: 06: 54$ \\
\hline & 4 & Occurring time of CGOB & & 19:04:31 \\
\hline
\end{tabular}

The total experiment process can be summarized as follows: coal sample production, coal packing and pressing, box sealing and installation, chamber air tightness test, coal inflatable adsorption, MS equipment installation, and press loading. During each experiment, the signals were recorded by the ESG MS equipment in real-time. The recorded events can be used to analyze the response and internal activities in the tested chamber. The rest of the detailed parameters of the process are referred to in the literature [40].

3.2. Experimental Process and Phenomena. The duration of coal outburst simulation parameters and result are summarized in Table 1, which corresponds to the test parameters and results of an experiment completed on December 25, 2011. The monitoring and analysis results indicate that the duration of the outburst process was approximately $15 \mathrm{~s}$. A total of $20.125 \mathrm{~kg}$ coal was ejected $(77.5 \mathrm{~kg}$ coal with $3.6 \mathrm{~kg}$ yellow mud infilling was installed). The key time points and parameters, including beginning time, occurring time, gas pressure, and other parameters, are shown in Table 1. Figure 5 reflects outburst situation at different times. Figure 5(d) displays the ejected coal in the outburst stage.

Responses during CGOB process were monitored by the MS system. Figure 6 shows the typical MS waveforms during the CGOB experiment. We can clearly observe the variation in MS signal in different moments, including amplitude and waveforms. For CGOB quantitative characterization, three aspects of the issues are discussed and validated in the next step.

\section{Time-Frequency Characteristic Analysis of MS Signals}

Mining MS signals possess typical nonstationary random and nonlinear properties. Time-frequency analysis is an important new branch of mathematics that has been recently developed. Time-frequency analysis has become of considerable research interest worldwide and thus has been used to analyze nonstationary MS signals.

4.1. Data Preprocessing. During the experiment, the vibration of loading device can trigger the MS monitoring system. The signal during CGOB was different from microcrack signal caused by coal and rock fracture. As the effect of gas, this kind of signal belonged to high-frequency vibration, and its frequency was higher than the original. In this experiment, the maximum frequency was close to $2,000 \mathrm{~Hz}$ (sampling frequency of $4,000 \mathrm{dBm} / \mathrm{Hz}$ ). Therefore, signal component 


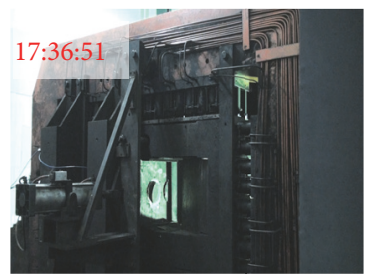

(a)

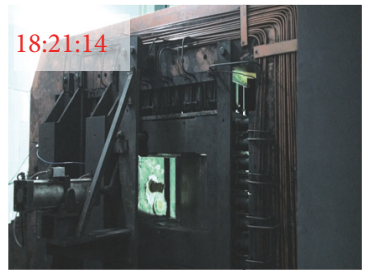

(b)

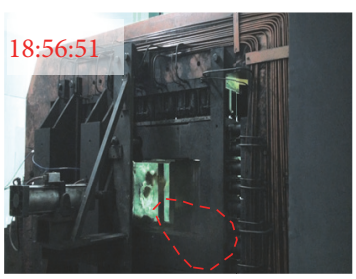

(c)

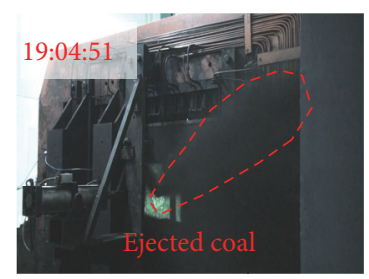

(d)

FIgUre 5: Phenomena of CGOB.
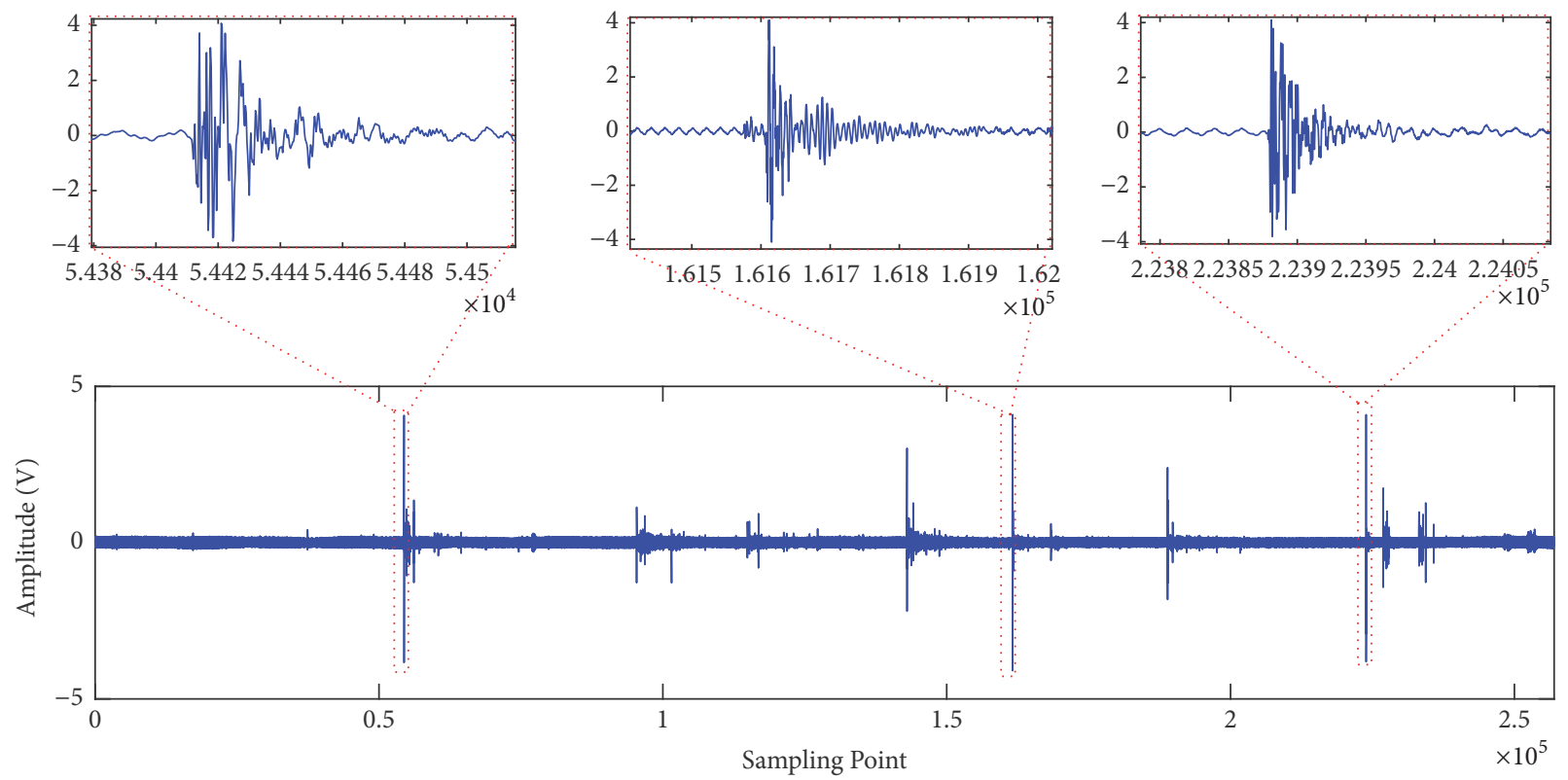

FIgure 6: Typical waveforms of MS signals in the CGOB laboratory.

distributed in the range of $0-100 \mathrm{~Hz}$ was not useful for analysis in this study and should be deleted before signal analysis.

Based on the comparison of several denoising methods, the notch filter was eventually elected to denoise MS signals. Figures 7(a1) and 7(b1) exhibit variations in the original waveform (Figure 7(a)) and the denoising waveform (Figure 7(b)) versus time, and Figures $7(\mathrm{a} 2)$ and $7(\mathrm{~b} 2)$ show the signal frequency of original and filter signal. Information of the original signal compared with Figure 7 shows that wavelet packet denoising better retained details and useful wavelet denoising. In addition, the processed signal was smoother than before.

4.2. MS Dynamic Response. The response frequency of the experiment was recorded at every $4 \mathrm{~min}$ during the burst process to highlight the features of the burst. The peak of the signal frequency $(\mathrm{Hz})$ versus time (min) (solid lines) and gas pressure $(\mathrm{MPa})$ versus time $(\mathrm{min})$ (solid lines with marker "o") are given in Figure 8. From the signal intensity of this figure, the distribution can be roughly divided into four stages, namely, incubation, inspire, happen, and residue stages, which agreed with the findings of $\mathrm{Hu}$ (2008) [10]. With the objective to observe the frequency change for a long period, the raw signal data were sliced to data pieces and data lengths of 4,000 sampling points (equal to 1 second) to mitigate the effects caused by data size. Figure 8 shows that the frequency distribution of MS signals scattered around is low in Stage I (event 1, almost less than $200 \mathrm{~Hz}$ ).

In Stage II (events 2 and 3), a general but slow increase in MS signal frequency of less than $400 \mathrm{~Hz}$ was observed with the gas pressure increase. The highest value of frequency in this stage can reach $632.68 \mathrm{~Hz}$. However, Stage III (events 4 and 5) deviated from Stages I and II. Large numbers of high-frequency signals emerged, and the signal frequency markedly changed from low $(0-400 \mathrm{~Hz})$ to high frequency (up to $1,600 \mathrm{~Hz}$ ). In Stage IV (event 6), calm was restored, and the frequency decreased to $50 \mathrm{~Hz}$ (power frequency). The variation curve of gas pressure matches the frequency change of MS signal. The CGOB exhibits significant periodic characteristics, and each CGOB stage corresponds to significant MS signal frequency.

This figure provides further evidence that the process of CGOB changes according to different stages along with a clear frequency change trend; that is, the variation trend in signal frequency moves from low to high.

4.3. Frequency Spectrum Analysis. According to Section 4.2, time-frequency analysis, an important method, was used for feature extraction of MS signal [35, 47]. This method 


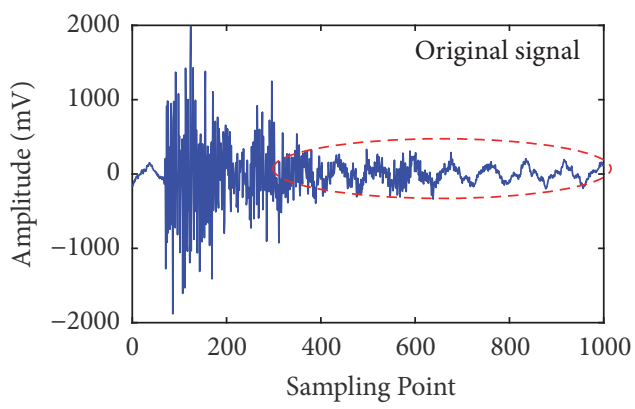

(a1)

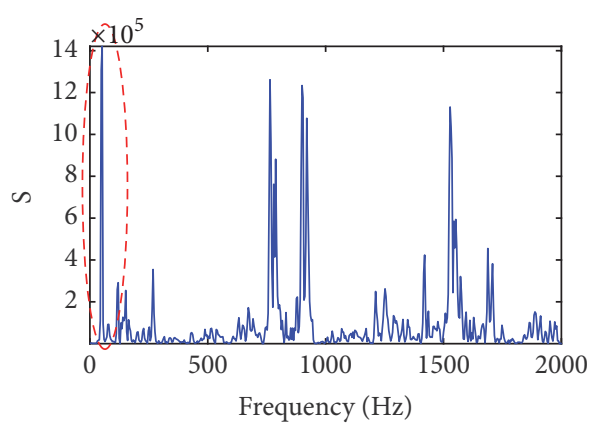

(a2)

(a) Original MS signal

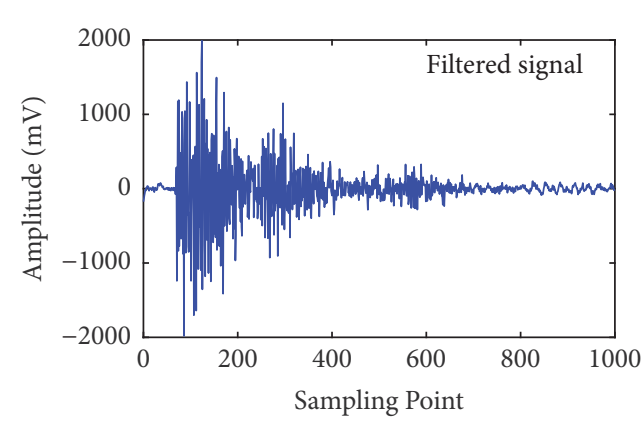

(b1)

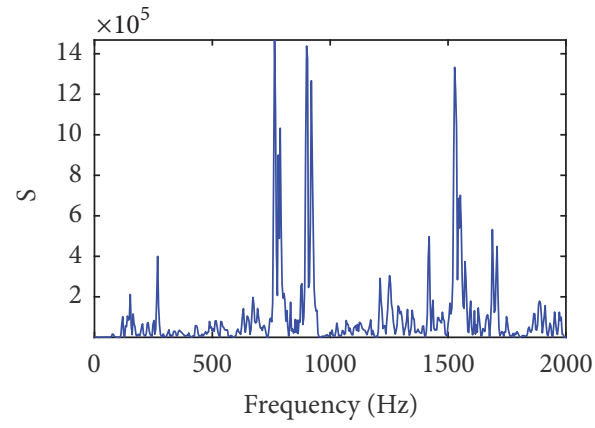

(b2)

(b) Filter MS signal

FIGURE 7: MS signal preprocessing results sampling point versus amplitude and frequency versus (a) original MS signal and (b) filtered MS signal.

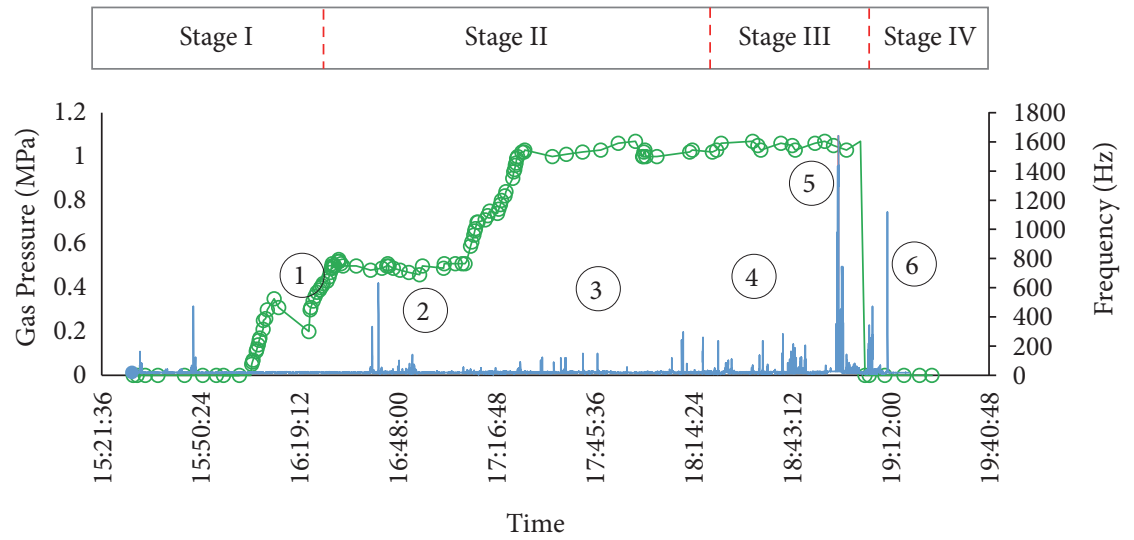

FIGURE 8: Variation in gas pressure levels and MS response (frequency) during the experiment.

can describe the relationship between time and frequency domains and provides conjoint distribution information of both sides (namely, energy density and intensity). Six typical events numbered 1-6 were detected from the CGOB experimental process and analyzed using short-time Fourier transform (see Figure 9) to mirror the microseismic signal's features in different stages.

Figure 9 shows that the distribution of frequency and energy with the variation in time and frequency can reflect certain characteristics. Events 1 and 2 occurred during Stage I. Event 1 mainly concentrated in $800 \mathrm{~Hz}$ with the subjective maximum value of $6,000 \mathrm{dBm} / \mathrm{Hz}$. This event was speculated as a mechanical vibration caused by experimental apparatus. Event 2 differed from 1 and 2 focused on $100-200 \mathrm{~Hz}$ and belonged to background noise. After a quiet period, event 3 occurred in Stage II with the increase in frequency (from 300 to $800 \mathrm{~Hz}$ ). CGOBs occurred in Stage III. Event 4 occurred with a series of MS events. Signal dominant frequency and energy reached $1,350 \mathrm{~Hz}$ (in the range $480-1,600 \mathrm{~Hz}$ ) and $1,800 \mathrm{dBm} / \mathrm{Hz}$ in this stage. Frequency was then distributed from 800 to $1,380 \mathrm{~Hz}$ and was concentrated at $850 \mathrm{~Hz}$ (see 5). After coal ejection, the frequency decreased to 50 

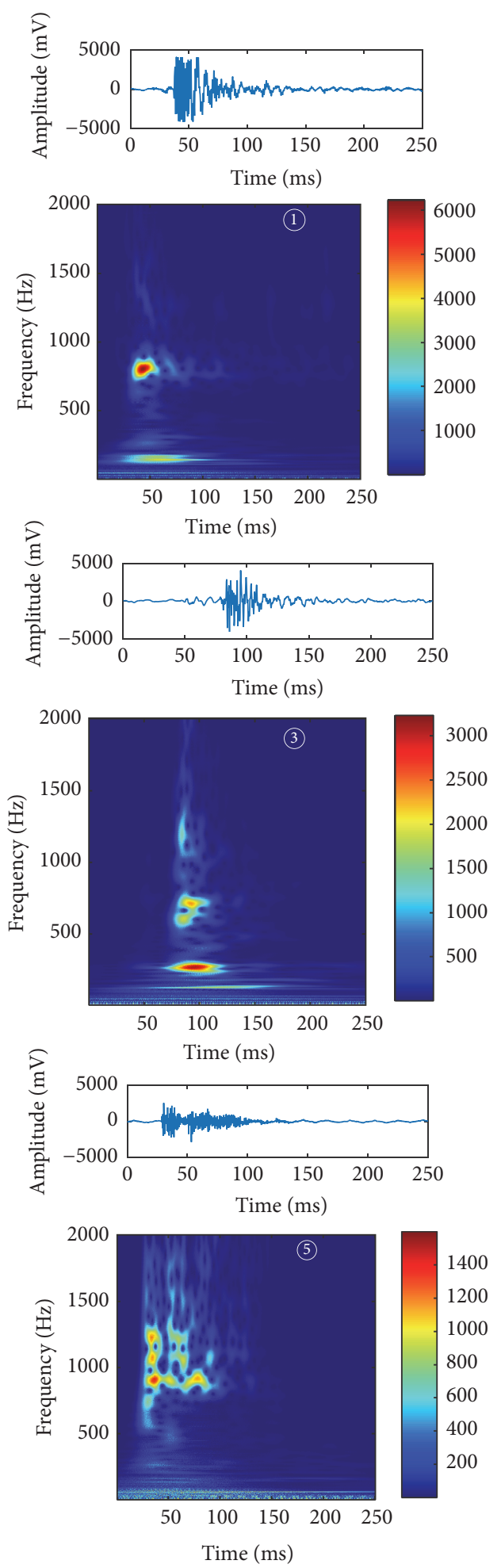
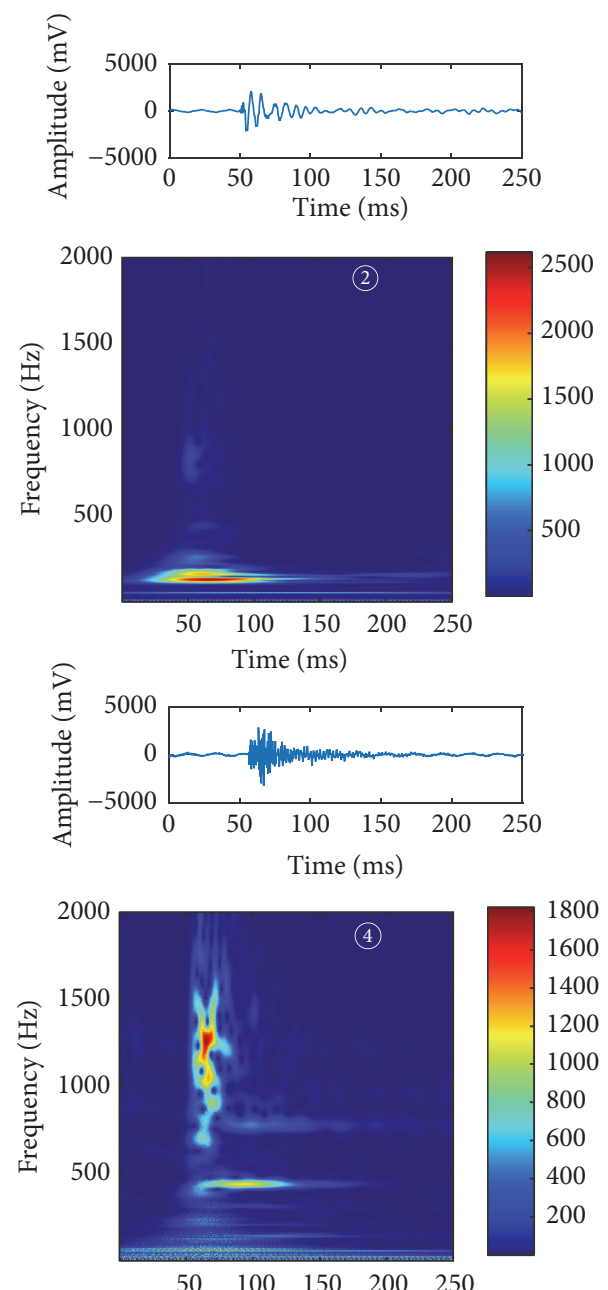

$\begin{array}{lllll}50 & 100 & 150 & 200 & 250\end{array}$
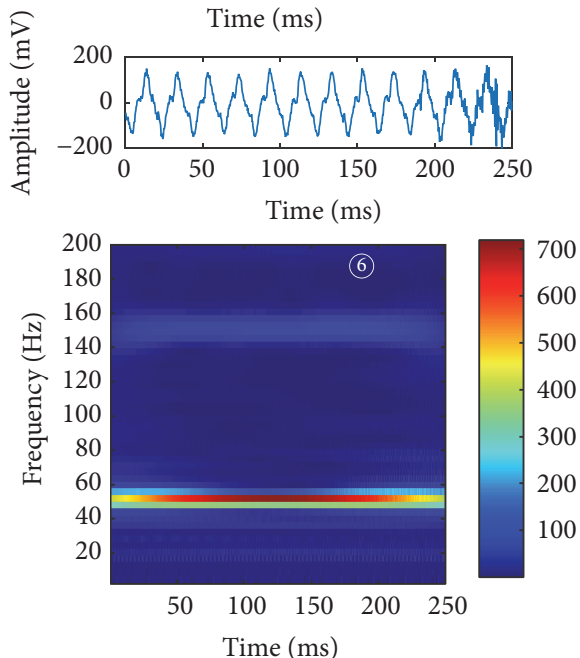

Figure 9: Evolution diagrams of MS signal spectrum under the CGOB test.

$\mathrm{Hz}$, and energy was $700 \mathrm{dBm} / \mathrm{Hz}$ at maximum (see 6). Comprehensive analysis shows no apparent changes in the energy and frequency of MS signals. In the outburst stage, the complicated frequency components gradually shifted from low to high frequency. This result validates the assumption in Section 2 . The remaining problem was how to quantitatively represent the MS signal and apply it in CGOB hazard prediction. 


\section{Prominent Signs of Seismic Energy Development}

5.1. Time-Frequency Analysis of Wavelet Packet Method. Wavelet packet transform (WPT), which can capture detailed information with fine resolution in both low- and highfrequency bands, was proposed by Wickerhauser [48] and Coifman [49] as generalization and improvement of wavelet transform. The WPT roughly divides the frequency space of a signal into a low-frequency and a high-frequency component by using a pair of low pass and high pass filters; the same splitting procedure is repeated for all obtained components until a specified level is reached. Through this process, the total frequency space of a signal can be divided into various narrow bands, allowing improved time-frequency localization and frequency components containing low energy to be readily identified [50]. This method exhibits good timefrequency characteristic, multiple scales, and multiresolution for confirming the features of MS signals. By assuming the MS signal is $s(t)$, wavelet packet decomposition model can be expressed as follows:

$$
s(t)=\sum_{n=0}^{2^{k}-1} s_{n}^{(j)}(t)
$$

where $s_{n}{ }^{j}(t)$ represents the $n$-th decomposed signal at $j$ layer scale.

Thus, the wavelet packet time-frequency component spectrum $W_{P} S_{\mathrm{n}}^{(j)}(\tau, f)$ is given as follows:

$$
\begin{gathered}
W P S_{\mathrm{n}}^{(j)}(\tau, f)=\int_{R} u_{n}^{[\mathrm{j}, 0]}(t-\tau) s_{n}^{(j)}(t) e^{-j 2 \pi f t} d t \\
=2^{-j / 2} \int_{R} u_{n}\left[2^{-j}(t-\tau) s_{n}^{(j)}(t) e^{-j 2 \pi f t} d t\right]
\end{gathered}
$$

The time-frequency component spectrum in $k$ layer and $j$ scale can also be expressed as

$$
\operatorname{WPS}^{(j)}(\tau, f)=\sum_{n=0}^{2^{k}-1} W P S_{\mathrm{n}}^{(j)}(\tau, f)
$$

Meanwhile, the time-frequency component spectrum accords with the conservation of energy. Hence, (3) can be expressed in Fourier transform method:

$$
\int_{R}|\mathrm{~s}(t)|^{2} \mathrm{dt}=\sum_{n=0}^{2^{k}-1} \int_{R} \int_{R}\left|W P S_{\mathrm{n}}^{(j)}(\tau, f)\right|^{2} d \tau d f
$$

With the help of the above equations, analysis of MS signals by using wavelet packet method becomes feasible.

5.2. Frequency Band Energy Calculation Method. Signal $s(t)$ (see (1)) can be decomposed into $2^{j}$ band components [39], forming $s_{0}^{(j)}(t), s_{1}^{(j)}(t), \ldots, s_{n}^{(j)}(t)$, and $s_{2^{j}-1}^{(j)}(t)$ because the MS signal is decomposed with wavelet packet, and the signal of each frequency band is reconstructed. The energy $E_{k}^{(j)}$ of component $s_{k}^{(j)}(t)$ is given as

$$
E_{k}^{(j)}=\int_{R}\left|s_{k}^{(j)}(t)\right|^{2} d t
$$

where $k \in\left\{0,1,2, \ldots, 2^{j}-1\right\}$.

The energy percentage of $k$-th component in the total original signal can be described as

$$
P_{n}^{(j)}=\frac{E_{k}^{(j)}}{E}=\frac{E_{k}^{(j)}}{\sum_{n=0}^{2^{j}-1} E_{n}^{(j)}}=\frac{\int_{R}\left|s_{k}^{(j)}(t)\right|^{2} d t}{\sum_{n=0}^{2^{j}-1} \int_{R}\left|s_{n}^{(j)}(t)\right|^{2} d t}
$$

5.3. Distribution of Wavelet Packet Energy. Wavelet packet analysis was applied to analyze the MS signal to extract the feature of stage signals. The basis function of wavelet package was "sym6". One channel signal was divided into 5 layers and 32 subbands, and every subband was $62.5 \mathrm{~Hz}$. Table 2 and Figure 10 show six events, and the distribution of band energy variation in time reflects the characteristics of four stages.

Table 2 and Figure 10 show apparent characteristics of frequency and energy variation, and frequency band energy distributions of MS signal largely differed from different stages during gas outburst. In this stage (before outburst), signal energy of number 2 is mainly distributed in the range of $187.5-250 \mathrm{~Hz}$ (S3), which accounts for up to $34.68 \%$ of the total signal. The same situation occurred in number 1. The distribution trend changes to high frequency along with the process. In the second stage (event 3), frequency composition is dispersed along a wide frequency range from S1 to S14. Signal energy increased to $12.75 \%$ in the S14 band. At the same time, bands S29 and S30 experience a modest increase of accumulated energy. The variation of frequency and energy distribution is apparent in events 4 and 5. The signal energy in this stage (outburst occurrence) distributes in a local area, such as S24-S30. In this frequency band range, total energy is $42.76 \%$ and $42.86 \%$ (average value $42.81 \%$ ), which are markedly larger than those of events $1-3(7.01 \%$, $(9.13 \%, 0.32 \%$, and $11.59 \%$, respectively)) and $6(1.44 \%)$. This phenomenon also shows the trend of signal frequency and energy transfer from low to high modes along with the process. Until the end of outburst (event 6, later period), the signal frequency changes and reverts to low frequency (S0).

For clearly showing the frequency band energy distribution of CGOB, MS signal versus time dependence was generated to obtain the characteristics of frequency band energy. Frequency band energy distribution of CGOB MS signals in different stages was clearly given in Figure 10, in which the high amplitude denotes large concentration and high energy of frequency. Signal change during the total process can be characterized as a progressive procedure involving "low frequency distribution-high-frequency transformationlow frequency rotation;" each step corresponded to "beforeduring-after" stages of the gas outburst. Therefore, the MS precursor characteristics of CGOB process can be excavated effectively by means of this quantitative method.

According to the above analysis, MS signal characteristics can be effectively obtained by the wavelet packet method. This method can provide detailed information by using wavelet 


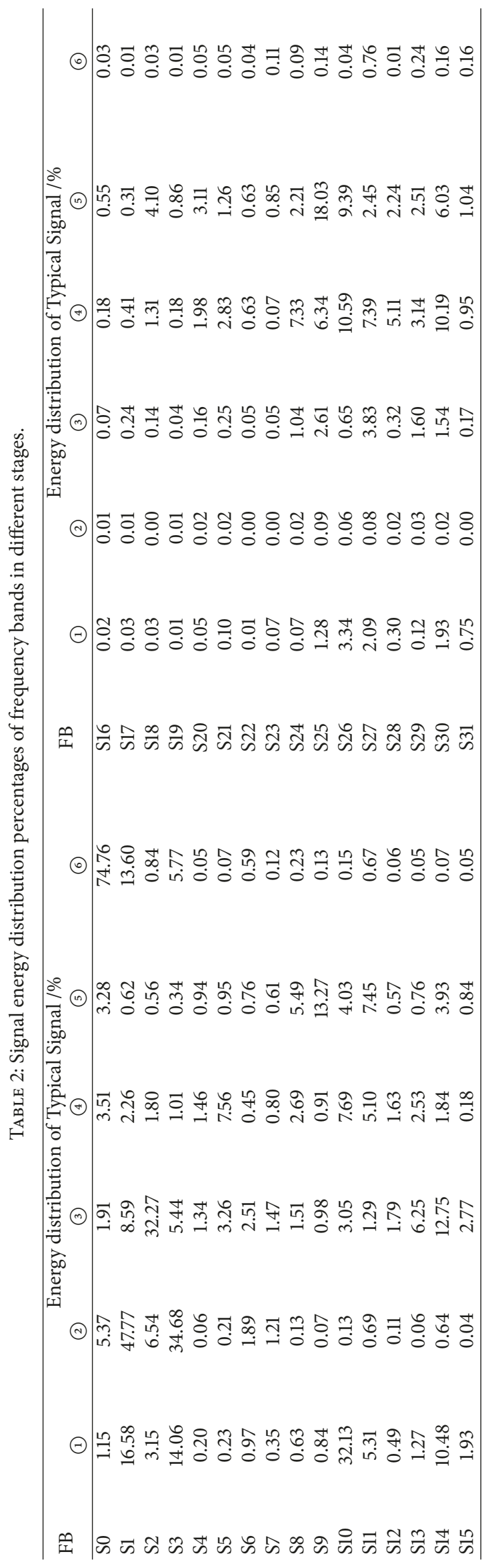



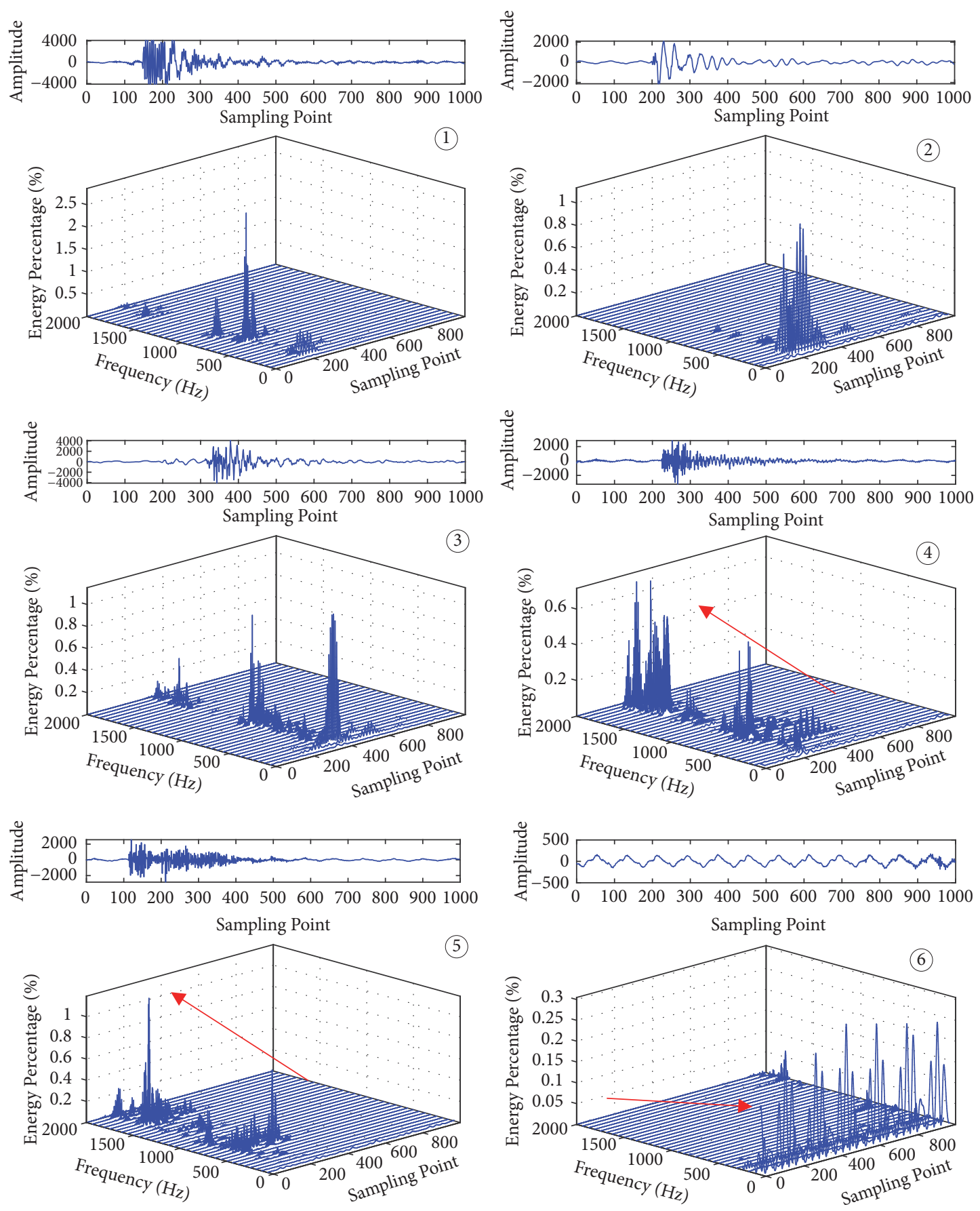

FIGURE 10: Frequency band energy distribution percentages of MS signals at different stages.

packet decomposition. Moreover, wavelet packet method can provide the frequency spectrum and energy characteristics of CGOB. Therefore, finding the precursor information of CGOB is crucial.

The discussion in this paper is only an initial conclusion, and numerous problems have to be solved until the goal of CGOB forecasting is achieved. Identification and intercept of valid waveform from MS real-time data and warning threshold value setting may be key issues in the future.

\section{Results and Discussion}

Through the above analysis, we obtain preliminary precursor and regularity of CGOB. This work is only the initial research about $\mathrm{CGOB}$ disaster prediction, and much remains to be done. The foreboding information is taken as prediction index for $\mathrm{CGOB}$, and a system of $\mathrm{CGOB}$ disaster prediction based on MS monitoring is proposed (Figure 11). The system is established based on the fundamental research about mechanism, influence factor of CGOB disaster, and 


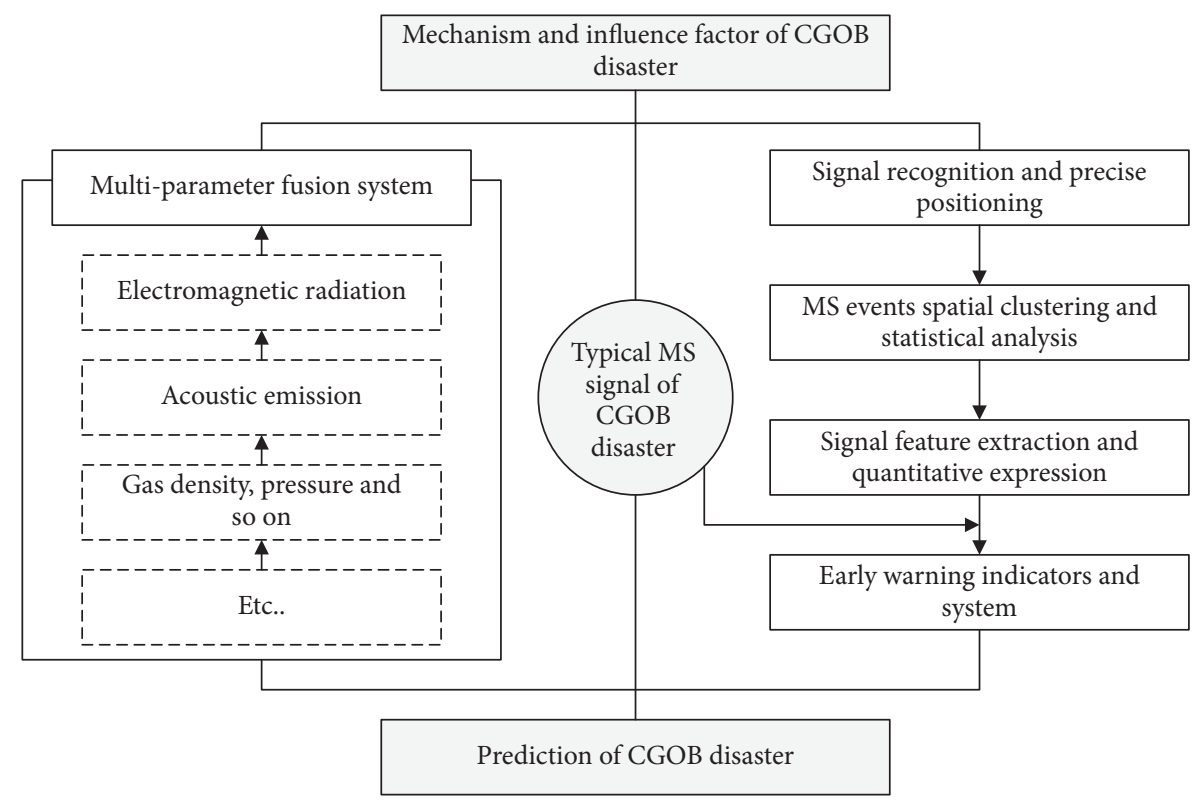

FIGURE 11: Prediction of CGOB disaster based on MS monitoring.

multiparameter monitoring, including MS, EMR, AE, and gas density and pressure. The principle of MS method uses feature mining and waveform recognition between monitoring data and typical MS signal of CGOB disaster. This research content includes several aspects, such as signal recognition and precise positioning, MS event spatial clustering and statistical analysis, signal feature extraction, quantitative expression, and early warning indicators and system.

\section{Conclusions}

Rockburst, especially the mixed disaster of coal and gas rockburst, is a challenging problem in coal mines that imposes serious danger to personnel and investment safety. Owing to its complexity associated with stress, gas, and physical and mechanical properties of coal, CGOB damage is difficult to forecast and control. Onsite monitoring and laboratory research indicate that microfracturing is a typical precursor of dynamic disaster, and MS technology has been widely used for mining disaster prediction. For this purpose, some CGOB experiments were carried out by means of a large CGOB physical simulation system with MS monitoring system.

Based on the MS monitoring system experiments and corresponding signal analysis and extractions, the conclusions of CGOB in this study can be drawn as follows. (1) The MS/AE behavior of coal failure experiment was studied first, followed by an explanation of laws: the fracture behavior of coal sample exhibits certain characteristics of MS/AE response in terms of $\mathrm{MS} / \mathrm{AE}$ event count, signal amplitude, and frequency, and each stage presents its own physical meaning during loading test. The precursor characteristics of AE signal/event can be a foundation of CGOB laboratory studies. (2) The approach presented in this study can capture dynamic response under CGOB testing. Through data preprocessing of MS signals, the method of wavelet packet transform is preferred in time-frequency, nonstationary outburst MS data, and even characteristic signal extraction. The features of each stage (incubation, inspire, happen, and residue stages), including the time-frequency domain, were extracted and quantitatively expressed. (3) Results of the CGOB MS data analysis showed that (1) the process exhibits its own MS response in different stages and represents using the time-frequency analysis method; (2) the frequency and energy density gradually transformed to highfrequency band (600-1,800 Hz), especially in $1,600 \mathrm{~Hz}$; (3) varying characteristics, such as "valley-peaks-valley," were observed in the total CGOB process.

Therefore, we assumed that the typical waveforms at different times in CGOB also exhibited different characteristics, especially the significant signal frequency changes before burst. If the assumption is reasonable, the quantization of CGOB signal characteristic, which is the basis of our present study, can be achieved.

The approach presented in this study can capture the dynamic response of a rock mass. The results of MS data analysis and interpretation provide dynamic response characteristics, changing characteristics, and a new idea for disaster prediction.

\section{Conflicts of Interest}

The authors declare that there are no conflicts of interest regarding the publication of this paper.

\section{Acknowledgments}

The authors thank the people who greatly helped in this work and everyone else who is involved with the project, including the stuff who participated in the laboratory. This study was funded by the National Natural Science Foundation of China 
(nos. 51604115 and 51504096), the Fundamental Research Funds for the Central Universities (3142017002), the Open Foundation of the Hebei State Key Laboratory of Mine Disaster Prevention (KJZH2017K14), the Technology Project and Self-Financing Foundation of Hebei Province (no. 16275512), and the Project of Langfang Key Technology Research and Development Program, China (Grant no. 2016013116).

\section{References}

[1] B. B. Beamish and P. J. Crosdale, "Instantaneous outbursts in underground coal mines: an overview and association with coal type," International Journal of Coal Geology, vol. 35, no. 1-4, pp. 27-55, 1998.

[2] L. Yuan, "Control of coal and gas outbursts in Huainan mines in China: A review," Journal of Rock Mechanics and Geotechnical Engineering, vol. 8, no. 4, pp. 559-567, 2016.

[3] C. Fan, S. Li, M. Luo, W. Du, and Z. Yang, "Coal and gas outburst dynamic system," International Journal of Mining Science and Technology, vol. 27, no. 1, pp. 49-55, 2017.

[4] J. Sobczyk, "A comparison of the influence of adsorbed gases on gas stresses leading to coal and gas outburst," Fuel, vol. 115, pp. 288-294, 2014.

[5] M. B. Wold, L. D. Connell, and S. K. Choi, "The role of spatial variability in coal seam parameters on gas outburst behaviour during coal mining," International Journal of Coal Geology, vol. 75, no. 1, pp. 1-14, 2008.

[6] S. Valliappan and Z. Wohua, "Role of gas energy during coal outbursts," International Journal for Numerical Methods in Engineering, vol. 44, no. 7, pp. 875-895, 1999.

[7] S. J. Peng, J. Xu, H. W. Yang, and D. Liu, "Experimental study on the influence mechanism of gas seepage on coal and gas outburst disaster," Safety Science, vol. 50, no. 4, pp. 816-821, 2012.

[8] W. C. Zhu, C. H. Wei, J. Liu, H. Y. Qu, and D. Elsworth, "A model of coal-gas interaction under variable temperatures," International Journal of Coal Geology, vol. 86, no. 2-3, pp. 213221, 2011.

[9] A. D. Alexeev, V. N. Revva, N. A. Alyshev, and D. M. Zhitlyonok, "True triaxial loading apparatus and its application to coal outburst prediction," International Journal of Coal Geology, vol. 58, no. 4, pp. 245-250, 2004.

[10] Q.-T. Hu, S.-N. Zhou, and X.-Q. Zhou, "Mechanical mechanism of coal and gas outburst process," Meitan Xuebao/Journal of the China Coal Society, vol. 33, no. 12, pp. 1368-1372, 2008.

[11] C. P. Lu, L. M. Dou, H. Liu, H. S. Liu, B. Liu, and B. B. Du, "Case study on microseismic effect of coal and gas outburst process," International Journal of Rock Mechanics and Mining Sciences, vol. 53, pp. 101-110, 2012.

[12] Y.-S. Pan, "Integrated study on compound dynamic disaster of coal-gas outburst and rockburst," Meitan Xuebao/Journal of the China Coal Society, vol. 41, no. 1, pp. 105-112, 2016.

[13] M. B. D. Aguado and C. G. Nicieza, "Control and prevention of gas outbursts in coal mines, Riosa-Olloniego coalfield, Spain," International Journal of Coal Geology, vol. 69, no. 4, pp. 253-266, 2007.

[14] J. Toraño, S. Torno, E. Alvarez, and P. Riesgo, "Application of outburst risk indices in the underground coal mines by sublevel caving," International Journal of Rock Mechanics and Mining Sciences, vol. 50, pp. 94-101, 2012.
[15] H. Zhou, R. Zhang, Y. Cheng, H. Dai, C. Ge, and J. Chen, "Methane and coal exploitation strategy of highly outburstprone coal seam configurations," Journal of Natural Gas Science and Engineering, vol. 23, pp. 63-69, 2015.

[16] B. Lin, F. Yan, C. Zhu et al., "Cross-borehole hydraulic slotting technique for preventing and controlling coal and gas outbursts during coal roadway excavation," Journal of Natural Gas Science and Engineering, vol. 26, pp. 518-525, 2015.

[17] V. Frid, "Electromagnetic radiation method for rock and gas outburst forecast," Journal of Applied Geophysics, vol. 38, no. 2, pp. 97-104, 1997.

[18] Y. Xue, F. Gao, Y. Gao et al., "Quantitative evaluation of stress-relief and permeability-increasing effects of overlying coal seams for coal mine methane drainage in Wulan coal mine," Journal of Natural Gas Science and Engineering, vol. 32, pp. 122$137,2016$.

[19] V. Frid, "Rockburst hazard forecast by electromagnetic radiation excited by rock fracture," Rock Mechanics and Rock Engineering, vol. 30, no. 4, pp. 229-236, 1997.

[20] L. M. Dou, H. E. Xq, and E. Y. Wang, "Electromagnetic emission technique of monitoring rock burst and its application," Journal of China Coal Society, vol. 29, no. 4, pp. 396-399, 2004.

[21] G. Si, S. Durucan, S. Jamnikar et al., "Seismic monitoring and analysis of excessive gas emissions in heterogeneous coal seams," International Journal of Coal Geology, vol. 149, pp. 4154, 2015.

[22] Y. Zhao, L. Liu, Y. Pan, B. Jiao, and C. Zhang, "Experiment study on microseismic, charge induction, self-potential and acoustic emission during fracture process of rocks," Yanshilixue Yu Gongcheng Xuebao/Chinese Journal of Rock Mechanics and Engineering, vol. 36, no. 1, pp. 107-123, 2017.

[23] Y. H. Zou, X. S. Zhao, and S. Liu, "Research on sound transmitted continued prediction technology for coal and gas outburst," International Journal of Coal Science \& Technology, vol. 33, no. 6, pp. 61-64, 2005.

[24] B. B. Gao, H. G. Li, H. M. Li, R. F. Yuan, and Y. P. Liu, "Current situation of the study on acoustic emission and microseismic monitoring of coupling dynamic catastrophe for gas-filled coalrock," Progress in Geophysics, vol. 29, no. 2, pp. 689-697, 2014.

[25] A. V. Shadrin, "Geophysical criterion of pre-outburst crack propagation in coal beds," Journal of Mining Science, vol. 52, no. 4, pp. 670-682, 2016.

[26] H. Ribeiro, L. Costa, L. Alves et al., "Analogies Between the Cracking Noise of Ethanol-Dampened Charcoal and Earthquakes," Physical Review Letters, vol. 115, no. 2, 2015.

[27] J. Dec and K. Pietsch, "A possibility of seismic identification of gas accumulations in Zechstein carbonate formations of the fore-sudetic monocline," Gospodarka Surowcami Mineralnymi / Mineral Resources Management, vol. 28, no. 3, pp. 93-112, 2012.

[28] L.-J. Dong, J. Wesseloo, Y. Potvin, and X.-B. Li, "Discriminant models of blasts and seismic events in mine seismology," International Journal of Rock Mechanics \& Mining Sciences, vol. 86, pp. 282-291, 2016.

[29] L. Dong, D. Sun, X. Li, and K. Du, "Theoretical and Experimental Studies of Localization Methodology for AE and Microseismic Sources Without Pre-Measured Wave Velocity in Mines," IEEE Access, vol. 5, pp. 16818-16828, 2017.

[30] L. J. Dong, W. Zou, X. B. Li, W. W. Shu, and Z. W. Wang, "Collaborative localization method using analytical and iterative solutions for microseismic/acoustic emission sources in the rockmass structure for underground mining," Engineering Fracture Mechanics, vol. 1, no. 32, 2018. 
[31] F. H. An and Y. P. Cheng, "An explanation of large-scale coal and gas outbursts in underground coal mines: The effect of low-permeability zones on abnormally abundant gas," Natural Hazards and Earth System Sciences, vol. 14, no. 8, pp. 4751-4775, 2014.

[32] E.-Y. Wang, X.-Q. He, Z.-T. Liu, and Z.-H. Li, "Study on frequency spectrum characteristics of acoustic emission in coal or rock deformation and fracture," Meitan Xuebao/Journal of the China Coal Society, vol. 29, no. 3, pp. 289-292, 2004.

[33] C.-P. Lu, L.-M. Dou, N. Zhang, J.-H. Xue, and G.-J. Liu, "Microseismic and acoustic emission effect on gas outburst hazard triggered by shock wave: a case study," Natural Hazards, vol. 73, pp. 1715-1731, 2014.

[34] G. Y. Zhao, Q. L. Deng, and J. Ma, "Recognition of mine microseismic signals based on FSWT time-frequency analysis," Chinese J. Geot. Eng, vol. 37, no. 2, pp. 306-312, 2015.

[35] Z. Yan, A. Miyamoto, and Z. Jiang, "Frequency slice wavelet transform for transient vibration response analysis," Mechanical Systems and Signal Processing, vol. 23, no. 5, pp. 1474-1489, 2009.

[36] X. Li, X. Shang, Z. Wang, L. Dong, and L. Weng, "Identifying Pphase arrivals with noise: An improved Kurtosis method based on DWT and STA/LTA," Journal of Applied Geophysics, vol. 133, pp. 50-61, 2016.

[37] R. Kimiaefar, H. R. Siahkoohi, A. R. Hajian, and A. Kalhor, "Seismic random noise attenuation using artificial neural network and wavelet packet analysis," Arabian Journal of Geosciences, vol. 9, no. 3, pp. 1-11, 2016.

[38] Y. Kang, W. Zhu, Q. Bai, L. Xie, and C. Tang, “Time-delay estimation of acoustic emission signals of rock using timefrequency energy analysis based on wavelet transform," Yanshilixue Yu Gongcheng Xuebao/Chinese Journal of Rock Mechanics and Engineering, vol. 29, no. 5, pp. 1010-1016, 2010.

[39] Q. Zhu, F. Jiang, Z. Yu, Y. Yin, and L. Lu, "Study on energy distribution characters about blasting vibration and rock fracture microseismic signal," Chinese Journal of Rock Mechanics and Engineering, vol. 31, no. 4, pp. 723-730, 2012.

[40] W. Lei, S. Li, P. Shang et al., "Coal and gas outburst simulation experiment reacted by microseismic monitoring," Caikuang yu Anquan Gongcheng Xuebao/Journal of Mining and Safety Engineering, vol. 31, no. 1, pp. 161-166, 2014.

[41] Q. J. Zhu, Q. S. Li, and S. Q. Li, "Microseismic dynamic response and characteristic analysis of coal and gas outburst experiment," Chinese Journal of Rock Mechanics and Engineering, vol. 31, no. 4, pp. 723-730, 2015.

[42] J. Zuo, J.-P. Pei, J.-F. Liu, R.-D. Peng, and Y.-C. Li, "Investigation on acoustic emission behavior and its time-space evolution mechanism in failure process of coal-rock combined body," Chinese Journal of Rock Mechanics and Engineering, vol. 30, no. 8, pp. 1564-1570, 2011.

[43] H. Zhao, G. Yin, H. Li, and Z. Wang, "Analysis on AE characteristic and its confining pressure effect of outburst coal containing gas," Chongqing Daxue Xuebao/Journal of Chongqing University, vol. 36, no. 11, pp. 101-107, 2013.

[44] S. D. Butt, P. K. Frempong, C. Mukherjee, and J. Upshall, "Characterization of the permeability and acoustic properties of an outburst-prone sandstone," Journal of Applied Geophysics, vol. 58, no. 1, pp. 1-12, 2005.

[45] G. Yin, H. Qin, and G. Huang, "Experimental study of characteristics of seepage and acoustic emission of gas-filled coal under different stress paths," Yanshilixue Yu Gongcheng Xuebao/Chinese Journal of Rock Mechanics and Engineering, vol. 32, no. 7, pp. 1315-1320, 2013.
[46] H. Ji and X. Lu, "Characteristics of acoustic emission and rock fracture precursors of granite under conventional triaxial," Chinese Journal of Rock Mechanics and Engineering, vol. 34, no. 4, pp. 694-702, 2015.

[47] C. P. Lu, L. M. Dou, X. R. Wu et al., "Frequency spectrum analysis on microseismic monitoring and signal differentiation of rock material," Chinese Journal of Geotechnical Engineering, vol. 27, no. 7, pp. 772-775, 2008.

[48] M. V. Wickerhauser, "Comparison of picture compression methods: wavelet, wavelet packet, and local cosine transform coding," in Wavelets: A Tutorial in Theory and Applications, pp. 679-700, Academic Press Professional, Inc., 1993.

[49] R. Coifman, Y. Meyer, and V. Wickerhauser, "Signal processing and compression with wavelet packets," in Wavelets and Their Applications, pp. 363-379, Springer, 1994.

[50] L. Law, J. H. Kim, W. Y. H. Liew, and S. Lee, "An approach based on wavelet packet decomposition and HilbertHuang transform (WPDHHT) for spindle bearings condition monitoring," Mechanical Systems and Signal Processing, vol. 33, pp. 197-211, 2012. 


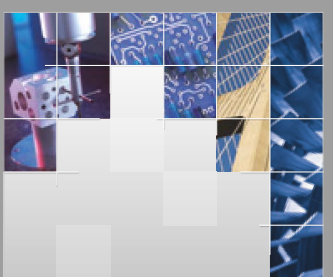

\section{Enfincering}
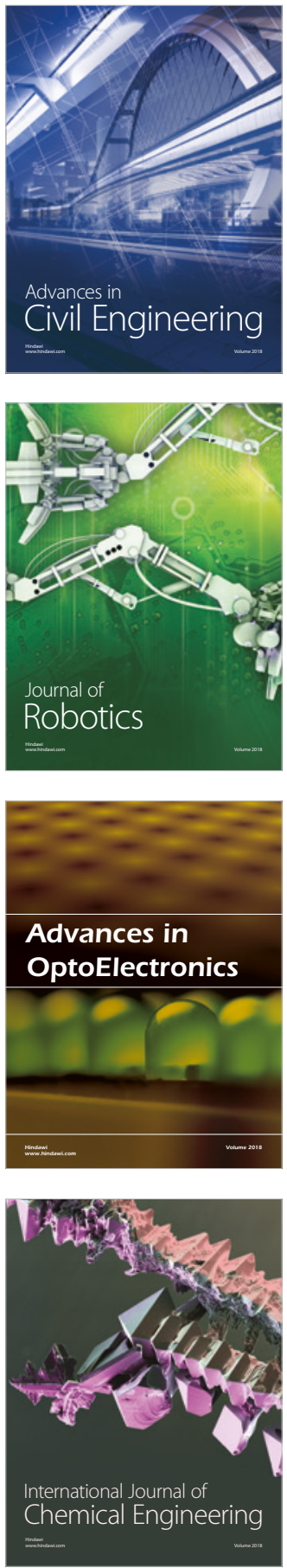

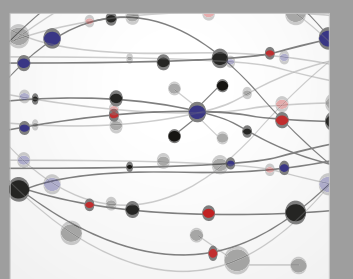

\section{Rotating \\ Machinery}

The Scientific World Journal

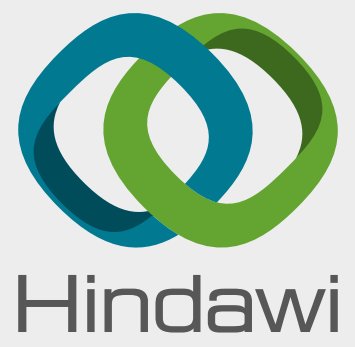

Submit your manuscripts at

www.hindawi.com
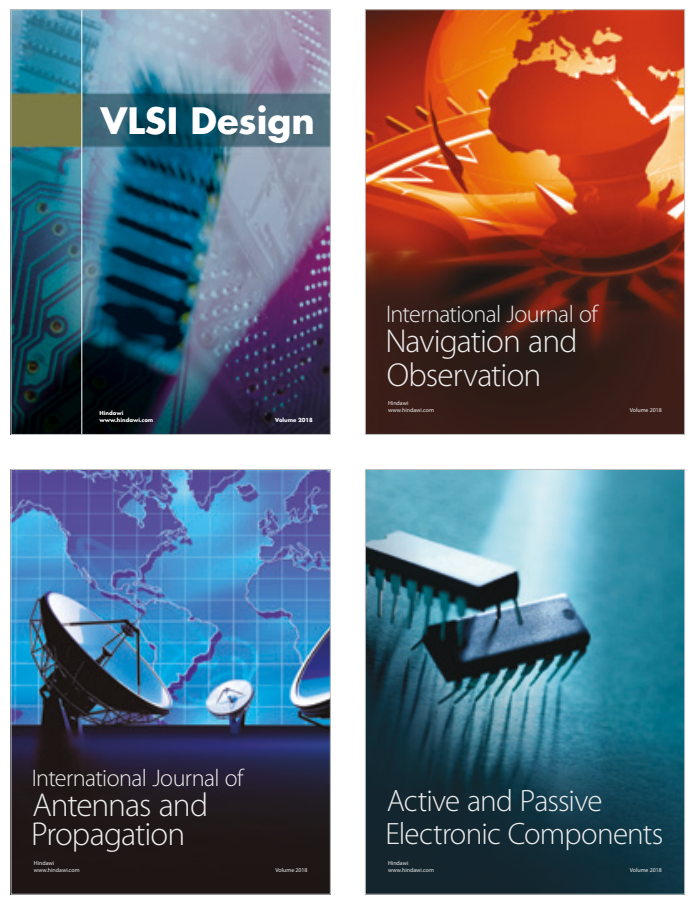
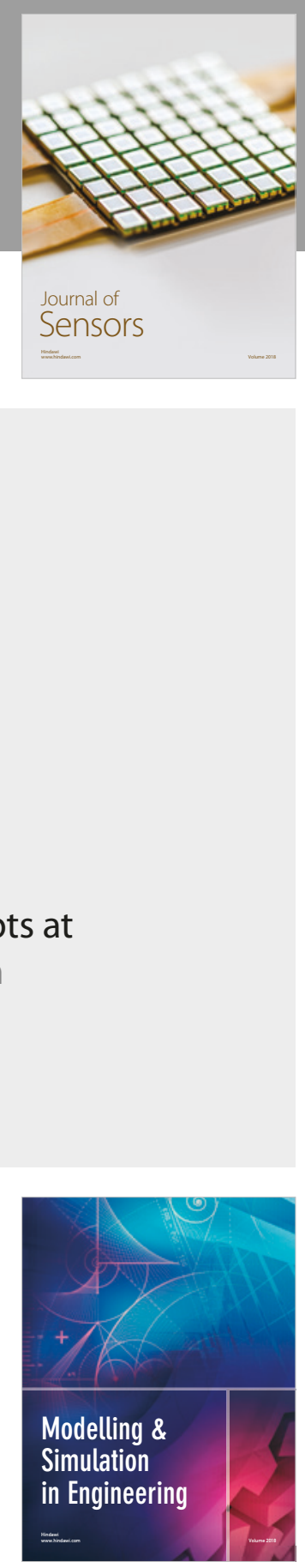

\section{Advances \\ Multimedia}
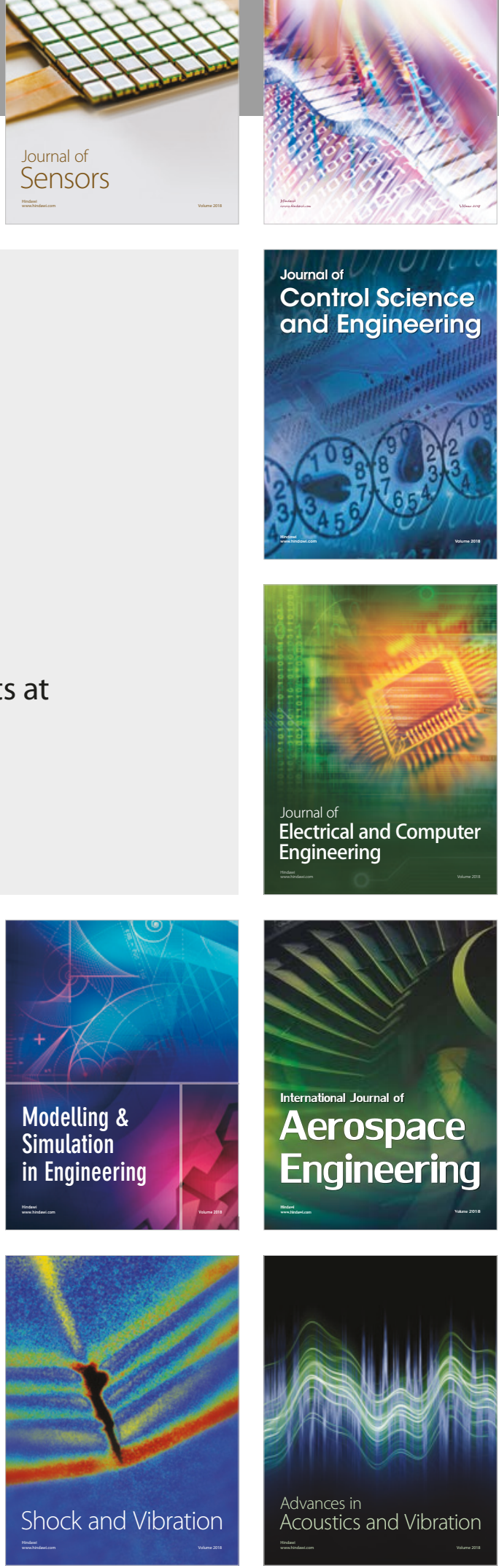